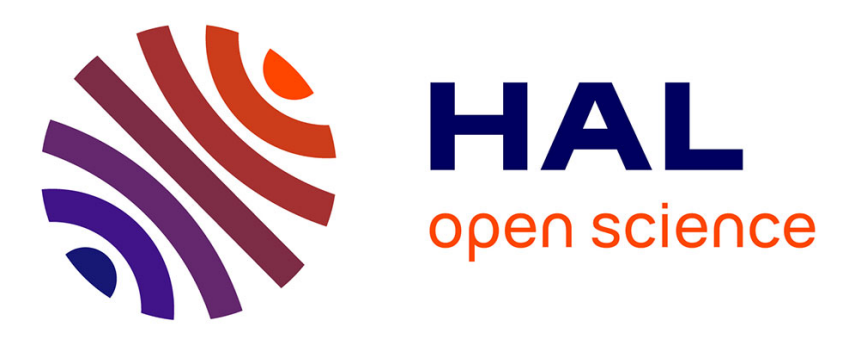

\title{
Magnetic and dynamic mechanical properties of a highly coercive MRE based on $\mathrm{NdFeB}$ particles and a stiff matrix
}

Svenja Hermann, Pauline Butaud, Gael Chevallier, Jean-François Manceau, Christophe Espanet

\section{To cite this version:}

Svenja Hermann, Pauline Butaud, Gael Chevallier, Jean-François Manceau, Christophe Espanet. Magnetic and dynamic mechanical properties of a highly coercive MRE based on NdFeB particles and a stiff matrix. Smart Materials and Structures, 2020, 29 (10), pp.105009 (11). hal-03360745

\section{HAL Id: hal-03360745 \\ https://hal.science/hal-03360745}

Submitted on 1 Oct 2021

HAL is a multi-disciplinary open access archive for the deposit and dissemination of scientific research documents, whether they are published or not. The documents may come from teaching and research institutions in France or abroad, or from public or private research centers.
L'archive ouverte pluridisciplinaire HAL, est destinée au dépôt et à la diffusion de documents scientifiques de niveau recherche, publiés ou non, émanant des établissements d'enseignement et de recherche français ou étrangers, des laboratoires publics ou privés. 


\title{
Magnetic and dynamic mechanical properties of a highly coercive MRE based on NdFeB particles and a stiff matrix
}

\author{
S Hermann ${ }^{1,2}$, P Butaud ${ }^{1}$, G Chevallier ${ }^{1}$, J-F Manceau ${ }^{1}$ and \\ C Espanet ${ }^{2}$ \\ ${ }^{1}$ Univ. Bourgogne Franche-Comté, FEMTO-ST, CNRS/UFC/ENSMM/UTBM, \\ Besançon, France \\ 2 Advanced Research and Development Unit, Moving Magnet Technologies SA, \\ Besançon, France. \\ E-mail: svenja.hermann@femto-st.fr
}

\begin{abstract}
This study concerns a magnetorheological elastomer (MRE) consisting of a magnetically hard powder and a stiff polymer matrix, so called STH-MRE. This material exhibits some strong constitutive differences with the behavior of MRE based on soft polymer matrix and magnetically hard powder (SOH-MRE). This paper presents a manufacturing process for such a STH-MRE, describes testing procedures to get the magnetic properties, the viscoelastic behavior and the magneto-mechanical coupling, and shares the STH-MRE properties with the scientific community. Firstly, the manufacturing process is detailed. Then, the homogenized magnetic properties and their dependence on the volume fraction and the temperature are discussed. Thereafter, dynamic mechanical analysis is explained and the viscoelastic properties are discussed considering the polymer matrix properties, the volume fraction and the temperature. Magneto-mechanical couplings are investigated in absence of an external magnetic field. The coercive field strenth of composite material and powder are almost equal while the remanent flux density depends on volume fraction. The temperature dependence of the magnetic properties is given as well as homogenization rules. The viscoelastic data of the STH-MRE are provided in a large temperature range and in the mechanical vibration frequency range. The magneto-mechanical coupling due to the remanent magnetization is shown to be negligible.
\end{abstract}

Keywords : Magnetorheological elastomer, H-MRE, magnetic hysteresis, dynamic mechanical analysis (DMA), magneto-mechanical coupling.

Submitted to: Smart Mater. Struct. 


\section{Introduction}

MagnetoRheological Elastomers (MRE) are an emerging type of active composite materials. They are composed of magnetic particles and a viscoelastic elastomer matrix and have thus interesting magnetic properties in addition to the mechanical ones. In mechanical engineering, elastomers are used as passive elements on account of their flexibility and resilience as well as for sealing and vibration damping purposes. When magnetic particles are embedded in an elastomer, some of the material properties become controllable by magnetic fields [1, 2], stiffness and damping properties can be modified for example. The performance of a MRE in an application is defined by its composition and its microstructure, hence the influence of parameters like filler content [3], particle size and arrangement in the matrix [3, 4] or matrix stiffness [5] have intensively been studied.

The elaboration procedure of an MRE generally begins with the dispersion of the particles in the matrix. In general, a degassing under vacuum is performed to avoid air bubbles in the mixture which can appear during the mixing of a liquid silicone and the powder. The mixture is molded and polymerization takes place during the curing step (see [6] for example). This elaboration procedure generates isotropic composites in which the particles are randomly dispersed. The second type of MRE structure of interest is a transversely isotropic arrangement of the particles in the matrix [7, 8, 9]. It is obtained by curing the composite in an external magnetic field which makes the particles form chains in the direction of the magnetic field lines.

Magnetically soft composites (S-MRE) generally contain particles that have a high magnetic permeability and saturation magnetization [4], carbonyl iron powder for example is used for the elaboration of S-MRE for these reasons. In an external magnetic field, the rigidity of S-MRE increases and their capacity to dissipate energy rises $[2,4,10]$. Transversely-isotropic composites are generally known for showing a stronger magnetorheological effect $[7,8]$ but it has been shown that this characteristic also depends on the particle size and distribution [4]. In absence of an external magnetic field, S-MRE do not show a significant magnetization.

To obtain a composite with permanent magnetization, magnetically hard materials like NdFeB are used [5, 11, 12]. Koo et al. published [13] one of the first papers on the characterization of the magnetic and mechanical properties of H-MRE based on various powders (barium ferrites, strontium, neodymium and samarium cobalt) and their use for actuators. The corresponding composite type is called H-MRE in the following. Recent studies show that the stiffness of H-MRE can not only be increased by external magnetic field but also be decreased $[7,14]$. This is mainly due to the particle motions and rearrangement in the polymer matrix, see $[13,15]$. To get this effect, the polymer matrix stiffness might be very soft, Young modulus $E \sim 10^{4}$ to $E \sim 10^{5} \mathrm{~Pa}$. In comparison with S-MRE, the use of H-MRE thus extends the adaptability of the material parameters. In the literature, a lot of attention has been paid to the active modification of the material parameters of soft matrix MRE under external magnetic 
fields due to particle rotations $[16,15]$. These studies include analysis of the viscoelastic behavior $[17,14]$ which show that the storage modulus and the loss factor are depending on the external magnetic field as well for the same reasons. They also show a strain rate dependence as well as a more commonplace frequency dependence.

In the present paper, the polymer matrix has been chosen stiff enough (Young modulus $E \sim 10^{6}$ to $\left.E \sim 10^{7} \mathrm{~Pa}\right)$ to avoid particle motions. This leads to another class of H-MRE, so-called ST(stiff)H-MRE. This is one the important novelty presented in this paper. Moreover, passive vibration control is targeted as an application for the STH-MRE using only the passive interactions, i.e. without external field. For these reasons, the material has to be studied from the magnetic and mechanical points of view but without external magnetic field, on the contrary to previous works. On the other hand, in order to explore the use of STH-MRE for the vibration control field, a specific attention has been payed onto the linear visco-elastic behaviour and its dependence on frequency and temperature. This knowledge is of great importance because a change in the excitation frequency changes the composite's stiffness and damping. Smart applications can take advantage of MRE for example in sandwich structures as shown by Szmidt et al. [18] who used an S-MRE and magnets. A STH-MRE could replace the S-MRE and the heavy magnets which is an advantage regarding the overall weight of the vibrating structure. Furthermore, magnetic interactions have recently been used for bandwidth enhancement in locally resonant metastructures [19]. The realization of magnetically polarized bistable structures for vibration canceling purposes is hence another application for STH-MRE.

The paper is organized as follows. Firstly, the manufacturing process for such a STH-MRE is completely detailed. Its micro-structure is then analyzed. Thereafter, the magnetic properties are discussed, dealing with the influence of filler content and temperature on the coercive field $H_{c}$ and the remanent flux density $B_{r}$. Afterwards the dynamic mechanical properties, storage modulus $E^{\prime}$ and loss factor $\tan (\delta)$, of the pure silicone matrix are compared with those of the composite. The influence of the magnetization on the dynamic mechanical properties is analyzed in the last section.

\section{Elaboration of an isotropic STH-MRE}

\subsection{Primary materials}

The STH-MRE which is used for the studies in this paper is composed of a magnetically hard powder and a silicone matrix. The elastomer MED-4014 [20] is provided by $\mathrm{NuSil}^{T M}$. It is a two-part silicone that cures with heat via platin catalyzed additioncure chemistry. The ratio of mixture of the two parts, $\mathrm{A}$ and $\mathrm{B}$, is 1:1. In the uncured state the consistency of the two parts can be compared to modeling clay. The magnetic powder MQFP-14-12 consists of NdFeB particles and is provided by Magnequench ${ }^{T M}$ in a non-magnetized state. The mean particle size $D 50$ is $(5.5 \pm 1.0) \mu \mathrm{m}$. According to the datasheet, the particles are magnetically isotropic, the remanent flux density $B_{r}$ of the 
material is $(805 \pm 20) \mathrm{mT}$ and the internal coercive field strength $H_{c}$ is $(965 \pm 55) \mathrm{kA} / \mathrm{m}$.

\subsection{Elaboration procedure}

To elaborate a STH-MRE with a specific volume fraction $\Phi$, the necessary mass of powder and silicone is calculated in a first step by the help of the mass density. The matrix material is not liquid in the uncured state, the dispersion of the particles in the matrix requires thus several mixing sequences. The procedure presented in the following is not an industrial process but allows the manufacturing of specimen with a simple geometric shape (figure 1). Of course, 3D printing should be investigated in the future, in order to get more complex geometrical shapes or magnetic patterns, see [21]. But, magnetization after polymerization is more favorable than the orientation of pre-magnetized particles during the material deposit if one seeks a high magnetization. Before the mixing begins, the silicone parts A and B are passed separately through a two-roll mill several times to soften. They are blended with the particles separately to prevent polymerization during the mixing. Once a part is softened, it is formed into a sheet. Afterwards a small quantity of powder is placed in the middle of the sheet and the silicone is then folded around the powder to enclose it. The mix is passed through the mill, folded again and milled again until the powder is evenly dispersed in the matrix. Small quantities of powder are added until the final volume fraction $\Phi$ is reached. The volume fractions of the composite specimen used for this study are comprised between $12.4 \%$ and $32.2 \%$. When both silicone parts have been prepared, they are milled together. The blended composite is placed into a mold. A pressure $p$ of $50 \cdot 10^{5} \mathrm{~N} / \mathrm{m}^{2}$ is applied on the mobile part of the mold to press the composite into its final form and evacuate eventual air bubbles. The curing is taken out under a temperature $T$ of $116^{\circ} \mathrm{C}$ during 10 minutes.

For the elaboration of MRE, a very viscous when uncured matrix is less often used [9] than a matrix that is liquid before polymerization $[5,6,7,8,11,14,21,22]$, probably because the mixing procedure is more complex. However, it offers the possibility to reach high volume fractions with lower risk of gravitation induced sedimentation of the particles during the time span of the elaboration.

For the present study, composite sheets of different thickness with an area of $200 \mathrm{~mm} \times 250 \mathrm{~mm}$ have been realized. The specimens are cut out via a cutting die or a conventional lathe. The magnetization is a supplementary step that is exhibited after the cutting process. A specimen is therefore placed in a strong external magnetic field of $4 \mathrm{~T}$ generated in a coil by a capacitor discharge and allowing the saturation of the NdFeB particles. When the external field is switched off, the composite retains a permanent magnetization since NdFeB is a magnetically hard material. The characteristics of all

specimens used in this study are given in the appendix, the references are given in the corresponding sections of this article. 


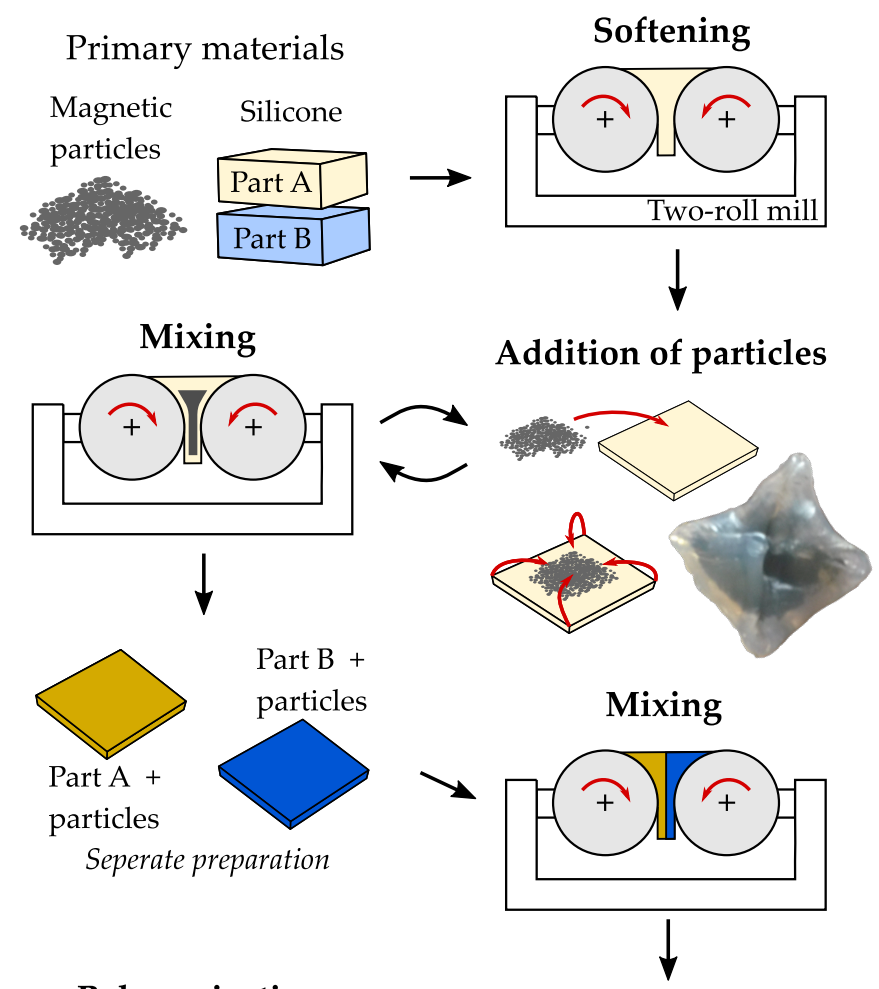

\section{Polymerization}
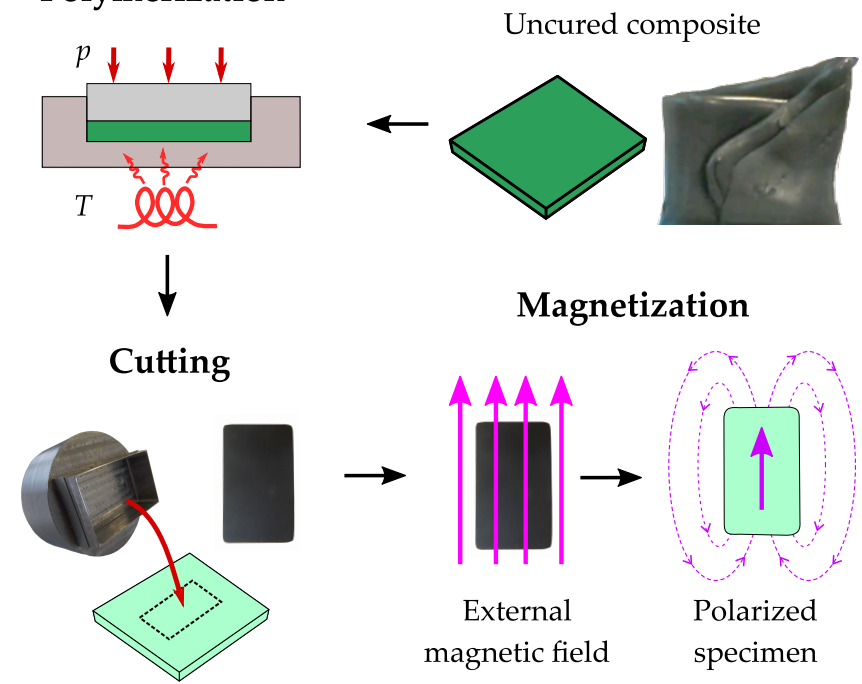

Figure 1. Elaboration procedure for magnetically hard polarized composite specimen

\subsection{Microstructure}

A Scanning Electron Microscope (SEM) is used to study the microstructure of an STHMRE with a volume fraction of $\Phi=19.4 \%$. The surface of observation lies in the cross section of the material and is obtained by freeze-fracturing. Figure 2a shows a SEM image of the fractured surface with 1000x magnification. The particles are denser than the silicone and reflect more electrons, thus they are the lighter spots in the image. They are dispersed randomly in the matrix and do not show a specific spatial orientation. This observation was also made for SEM images of surfaces lying in the 
three principal directions of the composite sheet. According to the SEM images, the composite is structurally isotropic. Figure $2 \mathrm{~b}$ shows a part of the fractured surface with 5000x magnification. A large size distribution as well as irregular forms of the particles are observed.

The analysis could only be taken out on non-magnetized samples as the magnetic field interferes with the electron beam of the SEM and the images gets blurred.

a)

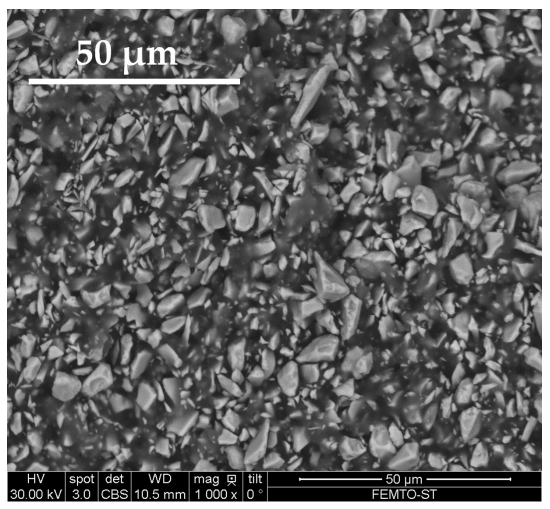

b)

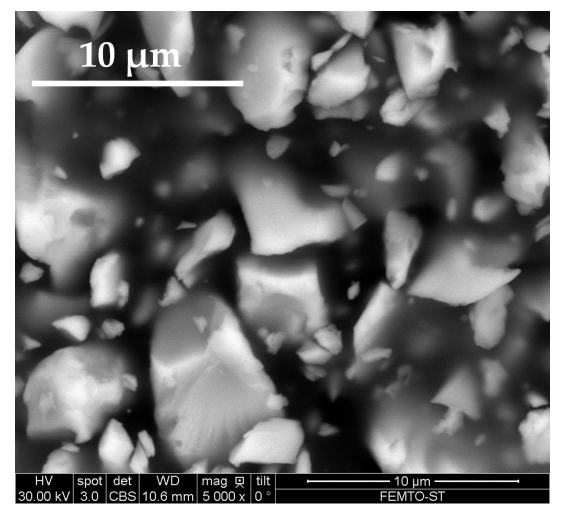

Figure 2. SEM image of a STH-MRE composite with a volume fraction of $\Phi=19.4 \%$ under 1000x (a) and 5000x (b) magnification.

\section{Magnetic characteristics}

\subsection{Demagnetization behavior and magnetic hysteresis}

In a first study, the dependence of the composite's magnetic polarization $J$ on the magnetic field $H$ is analyzed. Therefore, the demagnetization behavior of the magnetic powder is compared to the behavior of a composite material with a volume fraction of $\Phi=29.8 \%$ (specimen $S_{m a g 1}$, cf. Appendix, table 3). The measurement data of a compressed powder sample are provided by Magnequench while the composite specimen $S_{m a g 1}$ has been tested with an extraction magnetometer at the Institut Néel in Grenoble. The demagnetization behavior of the two samples is shown in figure 3a. At $H=0$, the remanent polarization $J_{r}$ (equivalent to the remanent flux density $B_{r}$ ) can be found. The polarization decreases under the influence of a magnetic field opposed to the magnetization of the samples. When the coercive field strength $H_{c}$ is reached, the material is demagnetized $(J=0)$. The comparison of the two materials shows that their coercive field strength $H_{c}$ differs slightly. It measures $969 \mathrm{kA} / \mathrm{m}$ for the powder and $941 \mathrm{kA} / \mathrm{m}$ for the composite, both values lie within the range specified for $H_{c}$ in the powder's datasheet. Their polarization $J_{r}$ differs much more: it measures $0.809 \mathrm{~T}$ for the powder sample and $0.227 \mathrm{~T}$ for the composite.

Figure $3 \mathrm{~b}$ shows the magnetic hysteresis cycle of two silicone-NdFeB composites having a volume fraction of $\Phi=29.8 \%$ and $\Phi=21.6 \%$ respectively (specimens $S_{m a g 1}$ and $S_{\text {mag } 2}$, cf. Appendix, table 3). The magnetic field was altered between $-6150 \mathrm{kA} / \mathrm{m}$ 
and $6150 \mathrm{kA} / \mathrm{m}$ during the tests with the extraction magnetometer. The observations made for the material behavior in the first tests shows again in the hysteresis cycles: the coercive field strength does not change but the remanent flux density $B_{r}$ does. It measures $0.174 \mathrm{~T}$ for the composite material with $\Phi=21.6 \%$. The dependence of $B_{r}$ on $\Phi$ will be discussed one of the following sections. Furthermore it can be seen from figure 3 that the magnetic hysteresis cycles of the two composite materials are symmetric. From these results it is concluded that the dilution of the powder in the silicone matrix affects the remanent flux density but not the coercive field of the studied composite material.

a)

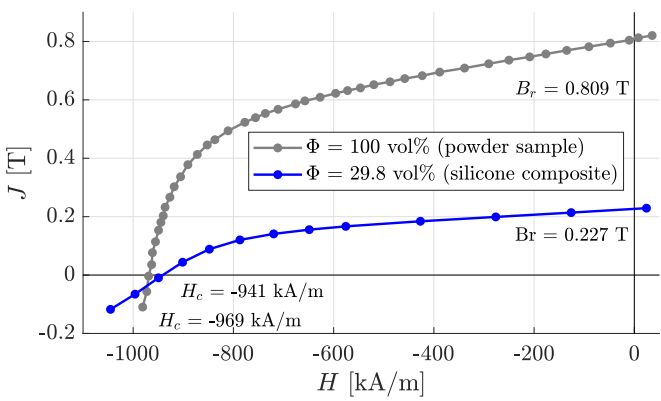

b)

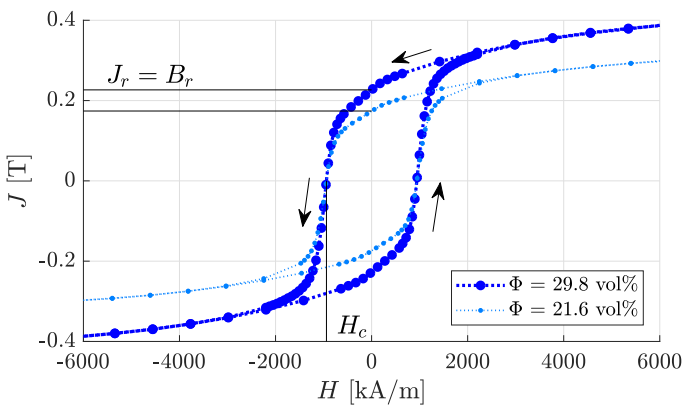

Figure 3. a) Demagnetization curves of a compressed powder sample and the specimen $S_{m a g 1}$ and b) magnetic hysteresis loop of the specimens $S_{m a g 1}$ and $S_{m a g 2}$ at $20^{\circ} \mathrm{C}$.

In previous studies on H-MRE, a significant difference of the coercivity between composites with a soft elastomer matrix ( $E$ in the range of $10 \mathrm{kPa}-30 \mathrm{kPa}$ ) and composites with a rigid matrix material has been observed in experimental analysis ([5]: PDMS and Epoxy, [14]: MRE above and beneath glass transition temperature) and as a result of modeling [23]. The origin of these differences caused by the movement of the particles in the matrix material, instead of turning the magnetic domains in the particle the particle itself rotates $[11,14,16]$. The rigidity of the matrix as well as the particle size and the volume fraction $[5,14,16]$ apparently have an influence on the mobility of the particles and consequently on the magnetic characteristics. In the present case the modulus of the matrix measures about $1 \mathrm{MPa}$ and the particles are relatively small $(D 50=(5.5 \pm 1.0) \mu \mathrm{m})$ which contributes to the presence of a high coercive field. The advantage of a high $H_{c}$ is a stronger resistance to demagnetization fields. Another indicator for the good fixation of the particles in the matrix is the symmetric hysteresis loop.

\subsection{Dependence on the temperature}

To study the influence of the temperature on the magnetic characteristics of a composite material with $\Phi=29.8 \%$, the magnetic hysteresis loop of the specimen $S_{m a g 3}$ (cf. Appendix, table 3) has been revealed for different temperatures ranging from $50{ }^{\circ} \mathrm{C}$ to $150^{\circ} \mathrm{C}$. Figure 4 shows the measurement results as well as the previous results for specimen $S_{m a g 1}$ recorded at $20^{\circ} \mathrm{C}$ since it contains the same volume fraction of particles 
as $S_{\text {mag3 }}$. Under rising temperature, the magnetic characteristics of the composite material are generally diminished. The magnetic polarization, and thus the intensity of the magnetic field of the composite material, decreases. At the same time, the coercive field strength decreases which means that the material can be demagnetized more easily.

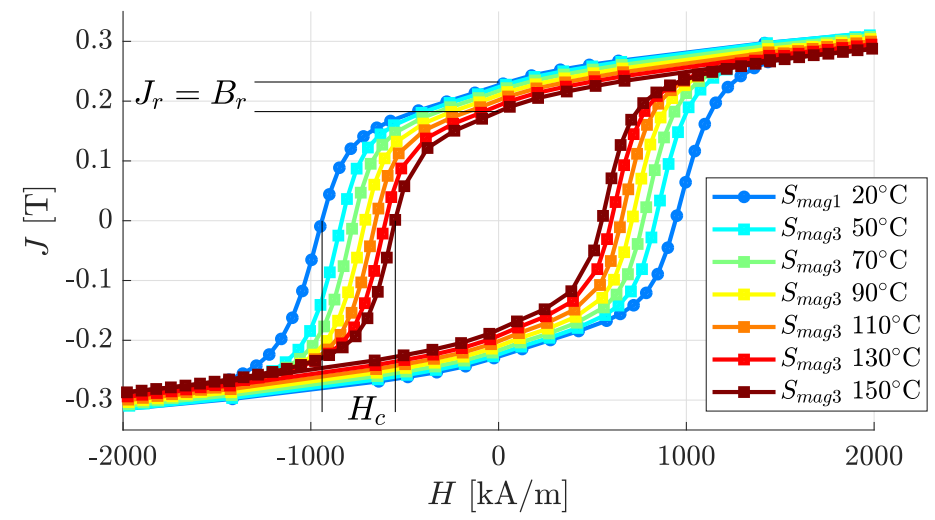

Figure 4. Magnetic hysteresis loops of the specimens $S_{m a g 1}$ and $S_{m a g 3}(\Phi=29.8 \%)$ at different temperatures.

The magnetic characteristics $B_{r}$ and $H_{c}$ of the powder are supposed decrease in a linear way with increasing temperature up to $100^{\circ} \mathrm{C}$. To estimate the decrease, the temperature coefficients $\alpha$ and $\beta$ are given in the datasheet. Starting from an initial temperature $T_{0}$, the characteristics of the powder at a temperature $T$ can be estimated by the following relations:

$$
\begin{array}{ll}
B_{r}(T)=B_{r}\left(T_{0}\right)+\alpha \cdot B_{r}\left(T_{0}\right) \cdot\left(T-T_{0}\right), & \alpha=-0.13 \% /{ }^{\circ} \mathrm{C}, \\
H_{c}(T)=H_{c}\left(T_{0}\right)+\beta \cdot H_{c}\left(T_{0}\right) \cdot\left(T-T_{0}\right), & \beta=-0.40 \% /{ }^{\circ} \mathrm{C} .
\end{array}
$$

To verify if this extrapolation can be applied the composite material as well, the measurement results of $S_{m a g 1}$ and $S_{m a g 3}$ are analyzed (figure 4). Equations 1 and 2 are used to estimate the evolution of the remanent flux density and the coercive field strength. $T_{0}=50^{\circ} \mathrm{C}$ is the initial temperature and the evolution of $B_{r}$ and $H_{c}$ is estimated in a temperature range from $20^{\circ} \mathrm{C}$ to $100^{\circ} \mathrm{C}$. Figure 5 a shows the remanent flux density measures for $S_{m a g}$ at $20^{\circ} \mathrm{C}$ and for $S_{m a g 3}$ at different temperatures between $50{ }^{\circ} \mathrm{C}$ and $150^{\circ} \mathrm{C}$. The corresponding values are listed in table 1 . The measurement error of $5 \%$ associated with the measurement values of $B_{r}$ is due to the specimen form and the resulting approximations on the demagnetization field. Therefore, an upper and lower bound of the estimation are shown in the graphic, they are calculated based on the upper and lower bound of $B_{r}\left(T=50^{\circ} \mathrm{C}\right)$. It can be seen, that the measurement values for $S_{m a g 3}$ are in good accordance with the estimation. Furthermore, the value measured for $S_{m a g 1}$, another specimen with the same volume fraction, at $20^{\circ} \mathrm{C}$ is in good accordance with the estimation as well. The temperature characteristics of the remanent flux density $B_{r}$ can be predicted with the help of the temperature coefficient of the powder up to $100^{\circ} \mathrm{C}$. 

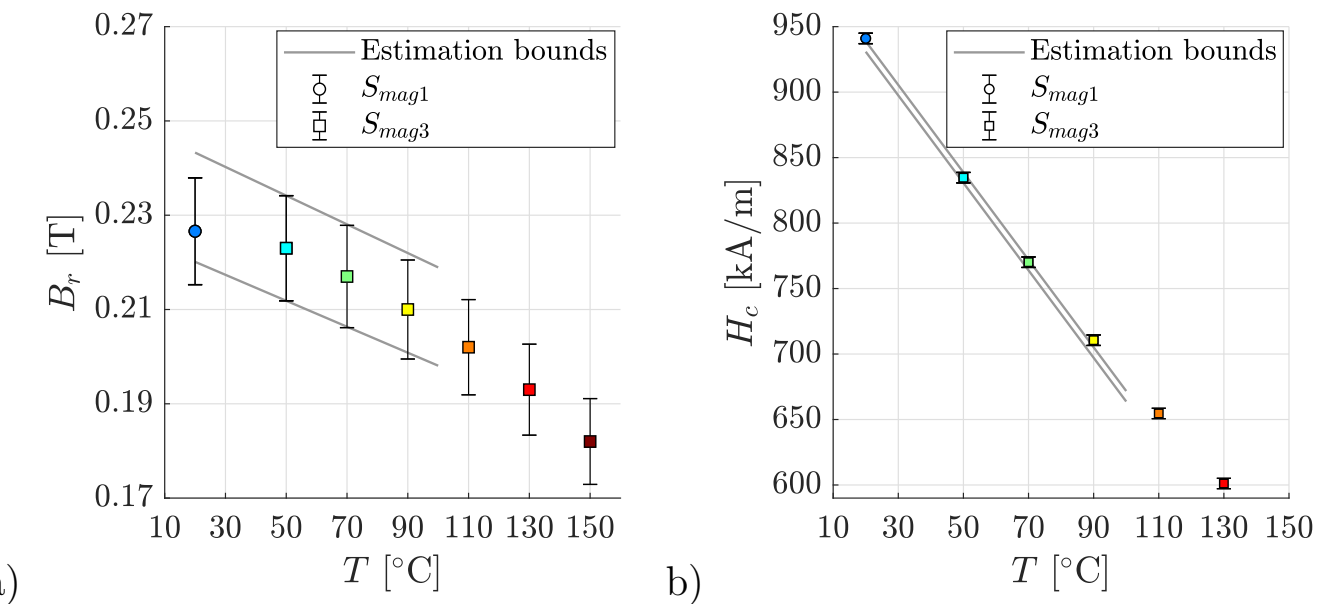

Figure 5. Dependence of remanent flux density $B_{r}$ (a) and coercive field strength $H_{c}$ (b) on the temperature, measurement values of $S_{m a g 1}$ and $S_{m a g 3}$ as well as estimation bounds.

Figure 5b shows the evolution of the coercive field strength $H_{c}$ of $S_{\text {mag3 }}$ between $50{ }^{\circ} \mathrm{C}$ and $90^{\circ} \mathrm{C}$. The measurement error of $4 \mathrm{kA} / \mathrm{m}$ is mainly due to the remanence of the extraction magnetometer. While the value for $H_{c}$ at $70^{\circ} \mathrm{C}$ is in good accordance with the estimation, the value at $90^{\circ} \mathrm{C}$ is slightly underestimated (cf. table 1 ). The coercive field strength of $S_{m a g 1}$ at $20^{\circ} \mathrm{C}$ on the other hand is in good accordance with the estimation. As the difference at $90^{\circ} \mathrm{C}$ measures only $2 \mathrm{kA} / \mathrm{m}$, it is concluded that the temperature characteristics of the coercive field strength $H_{c}$ can be predicted with the help of the temperature coefficient of the powder.

Table 1. $B_{r}$ and $H_{c}$ of $S_{m a g 1}$ and $S_{m a g 3}$ for different temperatures, LB - lower bound from estimation, UB - upper bound from estimation

\begin{tabular}{llllllcl}
\hline Specimen & $\mathrm{T}\left[{ }^{\circ} \mathrm{C}\right]$ & \multicolumn{2}{c}{$B_{r}[\mathrm{~T}]$} & \multicolumn{4}{c}{$H_{c}[\mathrm{kA} / \mathrm{m}]$} \\
& & $\mathrm{LB}$ & measurement & $\mathrm{UB}$ & $\mathrm{LB}$ & measurement & $\mathrm{UB}$ \\
\hline$S_{\text {mag } 1}$ & 20 & 0.226 & $0.227 \pm 0.011$ & 0.238 & 931 & $941 \pm 4$ & 939 \\
$S_{\text {mag3 }}$ & 50 & 0.217 & $0.223 \pm 0.011$ & 0.229 & 831 & $834 \pm 4$ & 839 \\
$S_{\text {mag3 }}$ & 70 & 0.212 & $0.217 \pm 0.011$ & 0.223 & 763 & $770 \pm 4$ & 712 \\
$S_{\text {mag } 3}$ & 90 & 0.206 & $0.210 \pm 0.011$ & 0.217 & 697 & $711 \pm 4$ & 705 \\
\hline
\end{tabular}

\subsection{Influence of the volume fraction $\Phi$}

As stated from the magnetometry tests, the volume fraction has an influence on the remanent flux density of the composite. To analyze this dependence more precisely, the remanent flux density of four isotropic composite materials with different volume fractions between $12.4 \%$ and $32.2 \%$ has been determined. Three specimens were cut out from each composite $\left(S_{m a g 4}-S_{m a g 15}\right.$, cf. Appendix, table 3$)$. Each specimen had a diameter of $5.5 \mathrm{~mm}$ and a height of $0.97 \mathrm{~mm}$. For the tests, the three magnetized 
specimens of each composite were stacked to form a cylinder with a height of $2.91 \mathrm{~mm}$ as for the measurement method described in the following, the geometric form of a long cylinder allows a more precise estimation of $B_{r}$ than flat pastilles. To approximate the remanent flux density, the voltage induced by the magnetic cylinders in a pair of Helmholtz coil has been measured. The specimen is placed in the coils and the voltage, that is induced in the coils by this procedure, is summed up by a voltage integrator. Knowing the geometry of the specimen, the number of windings, the resistance and the geometry of the Helmholtz coils as well as the electronic characteristics of the voltage integrator (capacity, resistance, amplification), the remanent flux density of the specimen is calculated. The four cylindrical samples were tested five times each and the mean values are represented in figure 6 (pink dots) and in table 2 . The standard deviation of the results due to the measurement method was smaller than $1.06 \%$ and is not shown in the graphic, but a deviation of $5 \%$ due to the specimen form and the resulting approximation on the demagnetizing field is indicated. As the relation between $B_{r}$ and volume fraction seems to be proportional, a linear regression of the measurement values has been done. The condition applied to the regression was an intersection with the origin of coordinates since a specimen without magnetic particles cannot be magnetized. A proportional factor of 0.0081 [T/\%] is obtained from the regression and the corresponding straight is shown as dashed line in figure 6 . The correlation coefficient is 0.9998 , the relation between remanent flux density and volume fraction is hence considered to be linear. A proportional correlation of the remanent flux density and the volume fraction can also be observed in the measurement results of previous studies $[14,21]$.

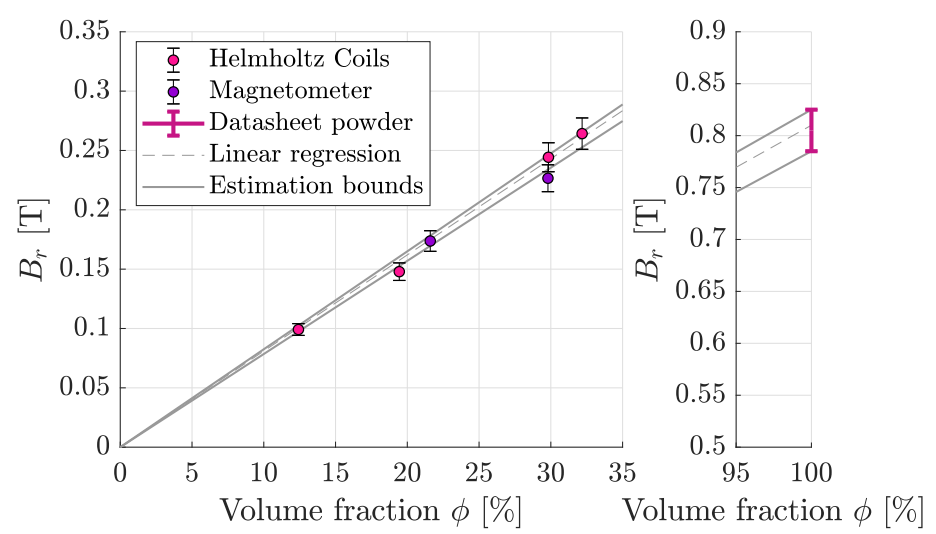

Figure 6. Dependence of the remanent flux density $B_{r}$ on the volume fraction $\Phi$.

Supposing a linear relation between remanent flux density and volume fraction, the possible values of $B_{r}$ of a STH-MRE can be limited by range given in the datasheet of the powder. In the present case, a minimum value of $0.785 \mathrm{~T}$ and a maximum value of $0.825 \mathrm{~T}$ are given (figure 6 , pink errorbar at $\Phi=100 \%$ ). The gray lines in figure 6 show the corresponding upper and lower bounds, limiting the values of $B_{r}$ for the composite. All measurement values lie in the range limited by these estimation 
Table 2. Remanent flux density as a function of the volume fraction

\begin{tabular}{rll}
\hline$\Phi$ & $\bar{B}_{r}$ & obtained by \\
{$[\%]$} & {$[\mathrm{T}]$} & \\
\hline 0.0 & 0.000 & linear regression : $B_{r}=\Phi[\%] * 0.0081$ \\
12.4 & 0.099 & measurement $S_{\text {mag } 4}-S_{\text {mag } 6}$ \\
19.4 & 0.148 & measurement $S_{\text {mag } 7}-S_{\text {mag } 9}$ \\
29.8 & 0.244 & measurement $S_{\text {mag } 10}-S_{\text {mag } 12}$ \\
32.2 & 0.264 & measurement $S_{\text {mag13 }}-S_{\text {mag } 15}$ \\
100.0 & 0.809 & measurement of powder sample \\
100.0 & 0.810 & linear regression: $B_{r}=\Phi[\%] * 0.0081$ \\
\hline
\end{tabular}

bounds. Furtheremore, the remanent flux density obtained from two magnetometry tests (purple dots) also lies within these bounds. The rule of mixtures can be used to find the remanent flux density of a homogeneous material equivalent to a composite with a specific volume fraction.

\section{Mechanical properties}

Silicone is a viscoelastic material, therefore, its dynamic mechanical properties are dependent on the time/frequency characteristics of the mechanical load. Dynamic mechanical properties are for example the storage modulus $E^{\prime}$ and the loss factor $\tan (\delta)$. In viscoelastic materials they do not only depend on the mechanical load but on the temperature as well [24]. Methods like the Dynamic Mechanical Analysis (DMA) allow the determination of the parameters necessary to identify the viscoelastic behavior laws [25]. In the present composite, the interactions between the particles and the matrix as well as particle-particle interactions can be added to the parameters that determine the mechanical behavior [26]. In this section, the results of a DMA on the pure matrix material and the non-magnetized composite material are presented.

\subsection{Dynamic Mechanical Analysis (DMA)}

Figure 7 shows the experimental setup of a DMA test in traction configuration. The specimens outer ends are fixed in two clamps. The upper clamp is fixed and connected to a force transducer measuring the reaction force $F(t)$. The lower clamp is mobile and a displacement $u(t)$ is imposed actuated by a shaker. The displacement control is done by a displacement transducer located above the shaker.

The harmonic displacement $u(t)$ is applied to the mobile clamp such as

$$
u(t)=u_{0}+u_{d} \cos (\Omega t),
$$

including a static displacement $u_{0}$ and a dynamic displacement $u_{d} \geqslant u_{0} / 2$. The reaction force $F(t)$ was measured at the fixed clamp such as

$$
F(t)=F_{d} \cos (\Omega t+\delta) .
$$



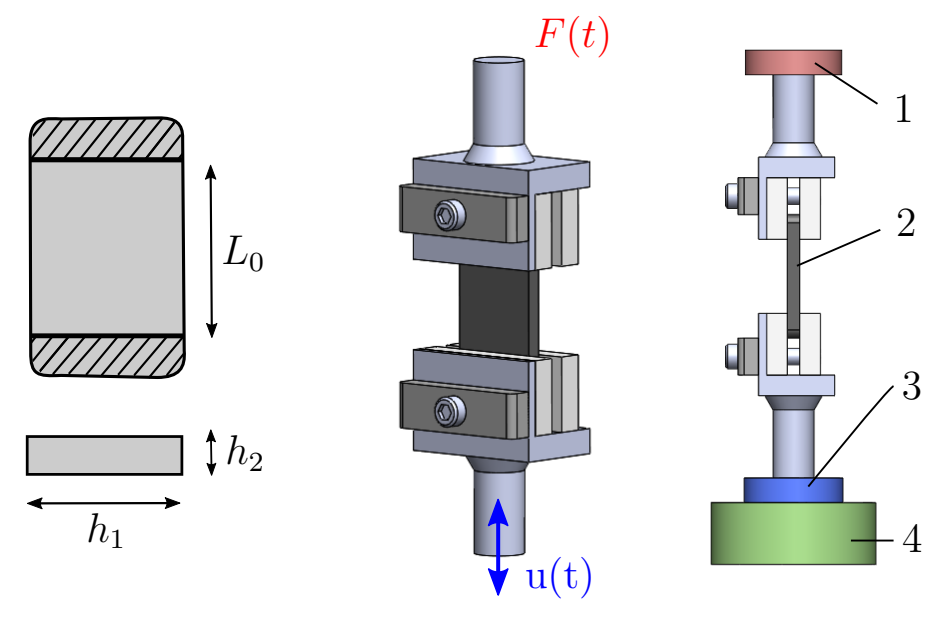

Figure 7. Geometric parameters of the DMA specimens and experimental setup containing 1-force transducer, 2-specimen, 3-displacement transducer, 4-shaker.

Figure 8 schematically depicts the force and displacement signals. The parameter $\delta$ describes the phase shift between displacement and force, the more viscous a material the bigger is the phase shift. The loss factor $\tan (\delta)$ is an indicator for the energy that is dissipated during the mechanical loading while the storage modulus $E^{\prime}$ is a measure of the ability of a material to recover from deformation. The two properties are described as follows:

$$
\begin{aligned}
& E^{\prime}=\frac{\bar{\sigma}}{\bar{\varepsilon}} \cos (\delta), \\
& \tan (\delta)=\frac{\bar{\sigma}}{\bar{\varepsilon}} \frac{\sin (\delta)}{E^{\prime}} .
\end{aligned}
$$

As it can be seen from the formulation a DMA is not a local measurement method but gives access to the average values of stress $\sigma_{d}$ and strain $\varepsilon_{d}$ in the specimen's volume $V$ :

$$
\begin{aligned}
& \bar{\sigma}=\frac{F_{d}}{h_{1} h_{2}}=\frac{1}{V} \int \sigma_{d} d V, \\
& \bar{\varepsilon}=\frac{u_{d}}{L_{0}}=\frac{1}{V} \int \varepsilon_{d} d V .
\end{aligned}
$$

All dynamic tests presented in the following are conducted with the dynamic mechanical analyzer DMA 1000 from Metravib in the configuration shown in figure 7 . The static and dynamic displacements were set to $u_{0}=5 / 1000 L_{0}$ and $u_{d}=1 / 100 L_{0}$ respectively. The displacement frequency ranged from $2.15 \mathrm{~Hz}$ to $100.00 \mathrm{~Hz}$. The DMA was performed for different temperatures ranging from $20^{\circ} \mathrm{C}$ to $150^{\circ} \mathrm{C}$, after each frequency scan the temperature was raised. Two specimens were tested for this study, a pure silicone specimen $\left(S_{m e c 1}\right)$ and a non-magnetized composite with $\Phi=19.4 \%\left(S_{m e c 2}\right)$.

Figure 9 shows the results obtained for $E^{\prime}$. In the given frequency and temperature range, the storage modulus of $S_{m e c 1}$ lies into the range from $1.29 \mathrm{MPa}$ to $1.78 \mathrm{MPa}$. 


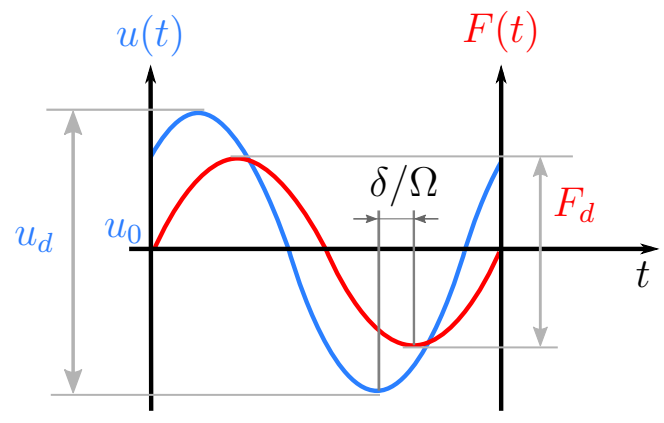

Figure 8. Schematic representation of force and displacement signals.

The maximum change caused by the temperature is of $11.8 \%$ and the chosen frequency range changes the modulus of $25.4 \%$ maximally. The storage modulus of $S_{m e c 2}$ lies into a range from $2.28 \mathrm{MPa}$ to $3.41 \mathrm{MPa}$, the composite is thus stiffer than the pure silicone. The temperature difference causes a maximum change of $16.4 \%$ and the frequency alters $E^{\prime}$ by $29.7 \%$. The storage modulus is slightly stronger influenced by the frequency and temperature changes as the pure silicone. The two materials show a behavior that is typical for elastomers in the rubbery state: the modulus increases with the frequency and a decreases with the temperature. The temperature enhances the chain mobility in the elastomer and the rigidity (respectively the modulus) decreases. Higher frequencies induce more elastic-like behavior of the silicone [27]. At low frequencies the viscous behavior predominates, the material flows.
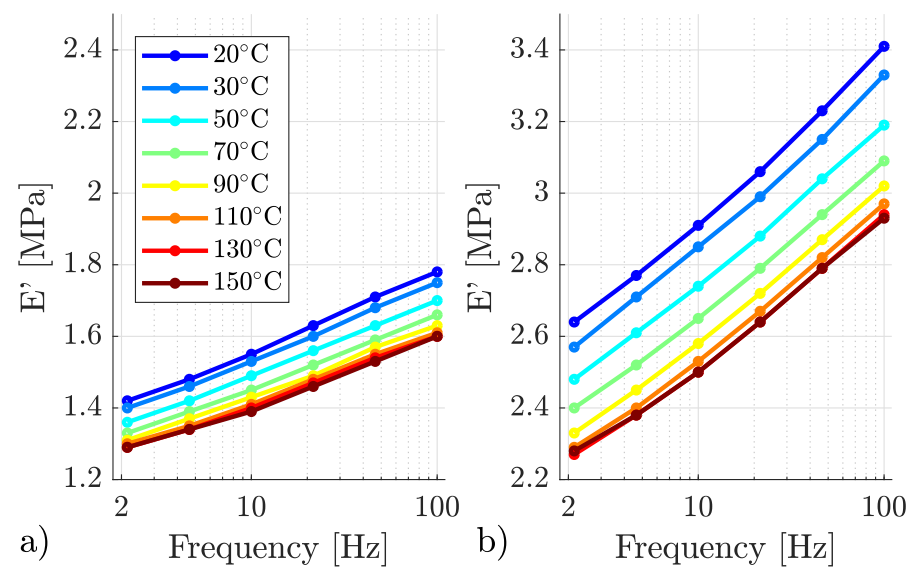

Figure 9. Storage modulus $E^{\prime}$ of the pure silicone specimen $S_{m e c 1}$ (a) and the composite $S_{\text {mec } 2}$ with $\Phi=19.4 \%$ in a frequency range from $2.15 \mathrm{~Hz}$ to $100.00 \mathrm{~Hz}$ and temperature range from $20^{\circ} \mathrm{C}$ to $150{ }^{\circ} \mathrm{C}$ at $1 \%$ deformation.

Figure 10 shows the loss factor of the silicone and the STH-MRE. For $S_{m e c 1}$ it lies in a range from $6.9 \%$ to $11.7 \%$ while $\tan (\delta)$ of $S_{m e c 2}$ reaches from $8 \%$ to $13.6 \%$. An increase in temperature decreases the loss factor. As the polymer chain mobility is increased by the temperature, they displace more easily and the energy dissipated by this process decreases, consequently the loss factor does the same. This behavior can be observed for 
both specimens while the magnitude of the loss factor is quite different. A continuous increase in frequency in the contrast does not have a monotonous effect even though an overall decrease of the loss factor from $2 \mathrm{~Hz}$ to $100 \mathrm{~Hz}$ is observed. In this range, the loss factor seems to decrease in steps for the pure silicone sample and even increase up to a frequency of $10 \mathrm{~Hz}$ at higher temperatures. The decrease in steps is less pronounced in the composite material but an increase in a frequency range up to $10 \mathrm{~Hz}$ is also observed at higher temperatures. For both materials, a temperature or frequency change in the given range can alter the loss factor more than $35 \%$.
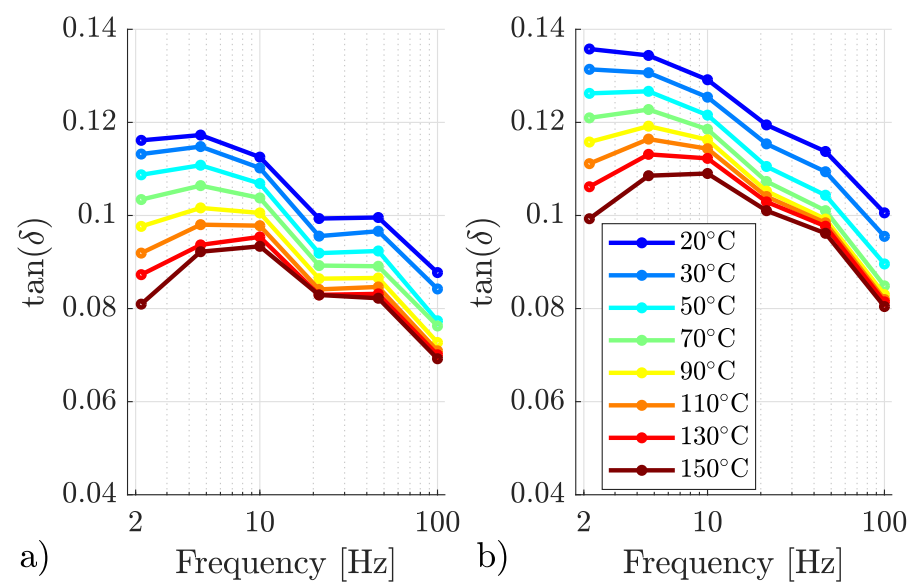

Figure 10. Loss factor $\tan (\delta)$ of the pure silicone specimen $S_{m e c 1}$ (a) and the composite $S_{m e c 2}$ with $\Phi=19.4 \%$ in a frequency range from $2.15 \mathrm{~Hz}$ to $100.00 \mathrm{~Hz}$ and temperature range from $20^{\circ} \mathrm{C}$ to $150{ }^{\circ} \mathrm{C}$ at $1 \%$ deformation.

Storage modulus and loss factor of the composite are significantly different from the ones of the silicone. Solicitation frequency and temperature however have in general the same influence on the mechanical characteristics of the two materials. Since the STH-MRE shows a viscoelastic behavior, two additional effects could be part of its mechanical behavior: the Mullins effect and the Payne effect. The Mullins effect is a damage induced and thus irreversible effect that emerges in the form of a decrease in rigidity. It appears for example during cyclic mechanical loading in filled elastomers [28]. The DMA tests were conducted in the so-called stabilized regime after some pre-cycling of the specimen. The Payne effect is the dependence of the dynamic modulus on the strain amplitude which is induced by nonlinear viscoelasticity above a certain strain threshold [29]. To avoid this effect as the study lies into linear viscoelasticity, the amplitude was kept small during the dynamic tests.

\section{Magneto-mechanical coupling}

In this section, the influence of a permanent magnetization of the STH-MRE on its mechanical characteristics is analyzed. On this purpose, the composite material has been subjected to a DMA with identical test configurations before and after magnetization. 
The eight non-magnetized specimens $S_{m m 1}-S_{m m 8}$ have been tested in a tensile configuration (figure 7) three times each. Between the three measurements, a specimen has been removed from the machine and mounted again. The strain amplitude was set to $1 \%$, the temperature to $T=30^{\circ} \mathrm{C}$ and the frequency to $f=1 \mathrm{~Hz}$. Afterwards, the specimens were magnetized. For half of the specimen the direction of magnetization was chosen parallel to their length and hence parallel to the mechanical solicitation $\left(S_{m m 1}\right.$ $\left.S_{m m 4}\right)$ which is shown schematically in figure 11b. For the other half, the direction was parallel to the specimen's thickness and perpendicular to the mechanical solicitation $\left(S_{m m 5}-S_{m m 8}\right)$, which is depicted in figure 11c. After the magnetization, each specimen was tested again three times. Due to the mounting of the specimen in the machine, the free length $L_{0}$ changed for each test. The corresponding values of $L_{0}$ for each specimen and test can be found in table 5 .

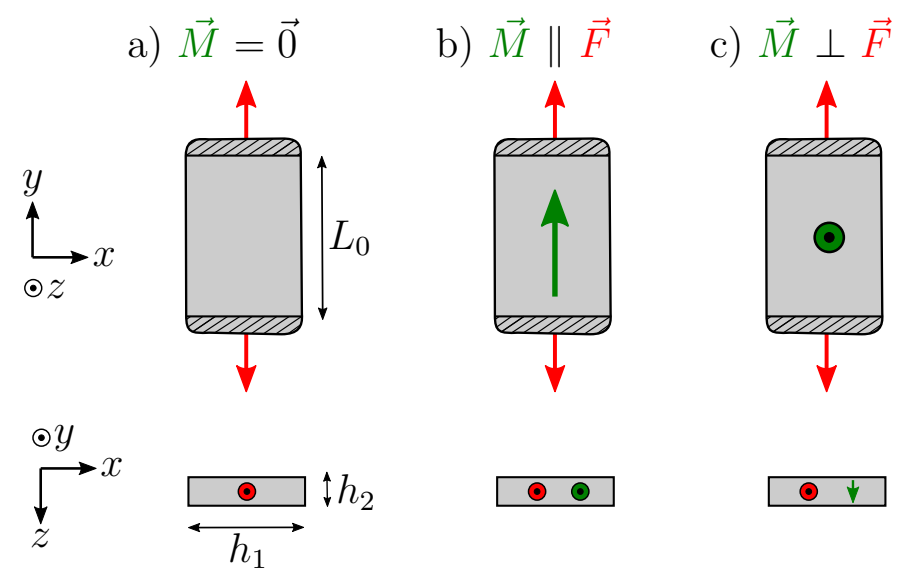

Figure 11. Scheme of the specimen in tensile DMA configuration (a) magnetized in the direction of its length (b) and its thickness (c).

To analyze the evolution of the dynamic mechanical properties before and after magnetization, relative values are compared. For each specimen the average of the three values of $E^{\prime}$ and $\tan (\delta)$ obtained before magnetization are calculated. All results are then divided by this mean. The relative values are called $E_{r e l}^{\prime}$ and $\tan (\delta)_{r e l}$ in the following. Figure 12 shows the mean relative storage modulus before and after magnetization for the eight specimens as well as the standard deviation of the three measurements for each specimen. On average, the modulus increases by $3 \%$ for $\vec{M} \| \vec{F}$, the tendency of an increasing modulus was found for all four specimens. The modulus decreases by $1.5 \%$ on average if $\vec{M} \perp \vec{F}$, the tendency of a decreasing modulus is also found for every tested specimen. However, both tendencies are not significant for all of the specimen.

Figure 13 shows the mean relative loss factor $\tan (\delta)_{\text {rel }}$ for the eight specimens before and after magnetization as well as the standard deviation of the three measurements for each specimen. The change of the mean loss factor does not show a clear trend like the change of the mean storage modulus. For $\vec{M} \| \vec{F}$ it increases for half of the specimen and decreases for the other half. For $\vec{M} \perp \vec{F}$ the trend is also not clearly visible since 

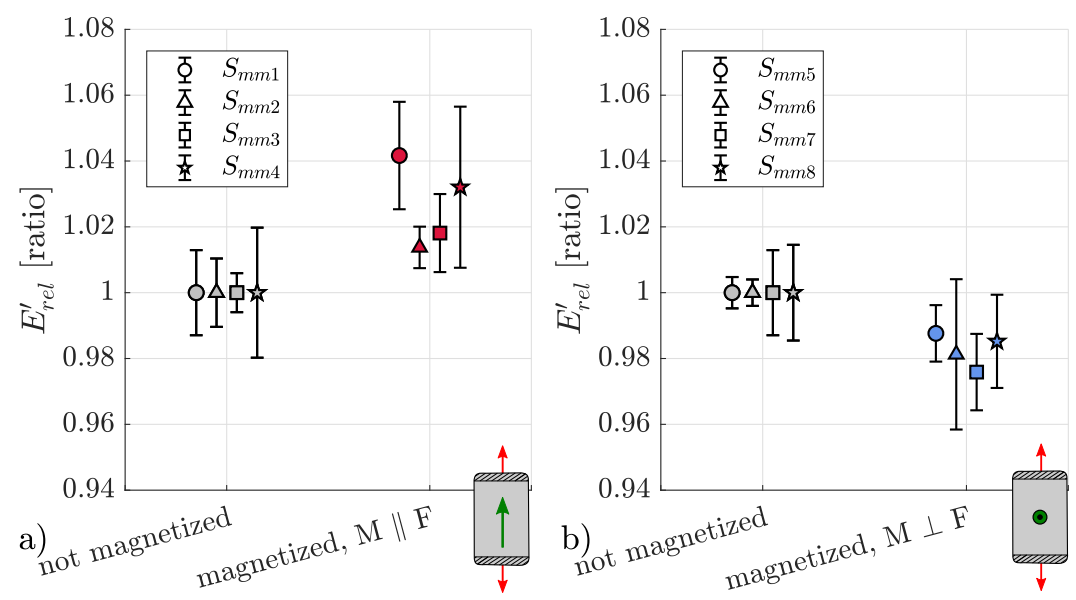

Figure 12. Relative storage modulus $E_{r e l}^{\prime}$ of the composite material before magnetization (gray) and after magnetization in the direction $\vec{M} \| \vec{F}$ (a) and $\vec{M} \perp \vec{F}$ (b).

$\tan (\delta)_{\text {rel }}$ decreases for one specimen and increases for the three other ones. On average, the loss factor increases by $0.3 \%$ when $\vec{M} \| \vec{F}$ and by $0.6 \%$ when $\vec{M} \perp \vec{F}$. Hence, the magnetization does not have a significant influence on the loss factor of the tested composite specimen. It has been noticed that the dispersion of the results increases strongly for the specimen magnetized in the direction $\vec{M} \| \vec{F}$, the increase is present but less pronounced if $\vec{M} \perp \vec{F}$.
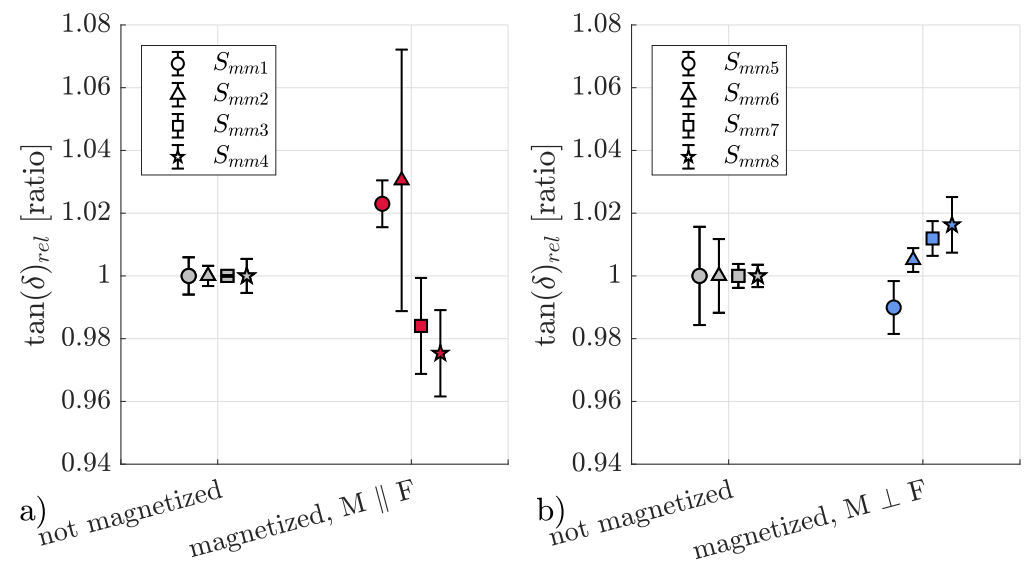

Figure 13. Relative loss factor $\tan (\delta)_{r}$ of the composite material before magnetization (gray) and after magnetization in the direction $\vec{M} \| \vec{F}$ (a) and $\vec{M} \perp \vec{F}$ (b).

Compared to previous analysis of the dynamic mechanical characteristics of SOH-MRE ([17]), the impact of the magnetization in this study is rather small. This is due to the matrix rigidity : the magnetic forces between the particles become small before the elastic forces of the matrix and hence the impact of the magnetization becomes small. Additionally, the impact of the geometric form of the specimen on the magnetic field distribution has to be considered in the analysis since the self-demagnetization of 
the specimen due to its form decreases the magnetic flux density inside the material. A numerical simulation of the specimens' self demagnetization in the air for the two magnetic states, $\vec{M} \| \vec{F}$ and $\vec{M} \perp \vec{F}$, has been done. In the simulation, $B_{r}$ was calculated from the characteristics of the powder and the volume fraction and hence set to $0.157 \mathrm{~T}, \mu_{r}$ was set to 1.1 based on the measurement results of specimen $S_{\text {mag } 1}$. The following analysis refers to the middle part of the specimen on which the measurement of the mechanical characteristics is based.

In the first case, where $\vec{M} \| \vec{F}$, the mean flux density in the material in the direction of $L_{0}$, called $B_{L_{0}}$ in the following, is of $0.151 \mathrm{~T}$ with a variation up to a maximum of $1.6 \%$. The specimen is magnetized in the direction of its longest extend $L_{0}$, this keeps the demagnetization field in the specimen small. Consequently, the magnetic flux density in the specimen does not decrease a lot compared to the remanent flux density $B_{r}$.

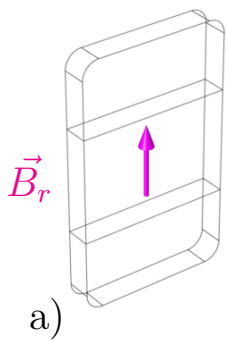

b)

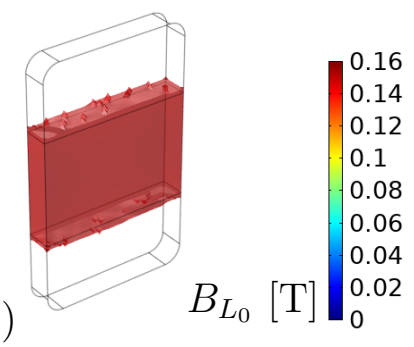

Figure 14. Numerical simulation of specimen's magnetic state in configuration $\vec{M} \| \vec{F}$ : a) direction of magnetization of the whole specimen, b) magnetic flux density $B_{L_{0}}$ in the mechanically loaded part of the specimen.

In the second case, where $\vec{M} \perp \vec{F}$, the mean flux density in the material is of $0.029 \mathrm{~T}$. The specimen is magnetized in the direction of its smallest extend $t$. The magnetic flux density in the direction of $t$, called $B_{t}$ in the following, is the highest on the outer bounds of the specimen where closing the magnetic flux lines is easier compared to the middle of the specimen. $B_{t}$ drops quickly with the distance towards the outer bounds and the variation within the evaluated section of the specimen is superior to $100 \%$. The magnetic flux density in the mechanically loaded part of the specimen is not homogeneous and the demagnetization effect causes a very low intensity of $B_{t}$ in most of the specimen section. The measurements results of this configuration are hence not representative in general for the composite material but are valid for this particular specimen geometry. 

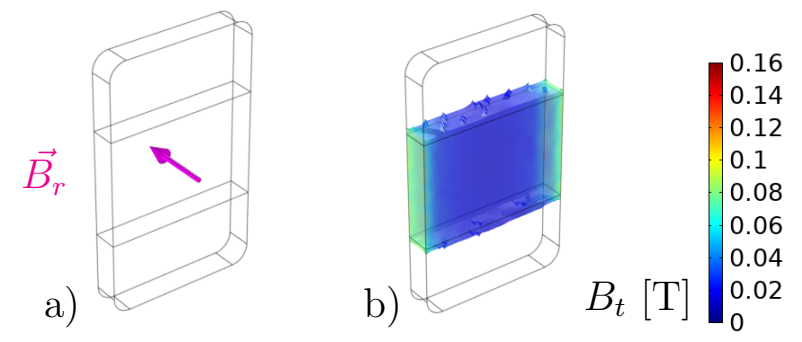

Figure 15. Numerical simulation of specimen's magnetic state in configuration $\vec{M} \perp \vec{F}$ : a) direction of magnetization of the whole specimen, b) magnetic flux density $B_{t}$ in the mechanically loaded part of the specimen.

\section{Conclusions}

In this work, isotropic STH-MRE composites were elaborated and the dependence of their magnetic and mechanical properties on different external and internal constraints was tested. It has been shown that, in absence of mechanical loading, the magnetic characteristics of the STH-MRE are determined by the powder. The temperature dependence of the remanent flux density and the coercive field can be predicted by temperature coefficients from the powder's datasheet. Furthermore, the remanent flux density has been shown to be a linear function of the volume fraction. The dynamic mechanical analysis shows that an increase of the frequency as well as a decrease in temperature increase the material rigidity. The energy dissipation rises when the temperature is decreased. A significant change of the dynamic mechanical properties due to the magnetization of the STH-MRE has not been observed. The magnetomechanical coupling in absence of an external magnetic field is too weak to have an effect on the material behavior of the composite specimen used in this study. The design of smart structures using STH-MRE has to take into account the dependence of the composite's properties to the temperature and the frequency of mechanical solicitation. The influence of the remanent magnetization on the macroscopic mechanical properties $E^{\prime}$ and $\tan (\delta)$ seems to be less significant in absence of an external magnetic field but has to be analyzed precisely in future studies. The measurement technique to reveal a coupling can be based on average quantities, in this case the geometry of the specimen has to be adapted to create a homogeneous internal magnetic field. Full field measurement techniques like digital image correlation on the other hand could be helpful for analyses of structures other than ellipsoids or long cylinders. Besides tests in an external magnetic field and the study of nonlinear mechanical behavior, another interesting topic for future studies is the impact of a mechanical solicitation on the internal magnetic state of a STH-MRE. 


\section{Acknowledgements}

This work has been funded by the french company Moving Magnet Technologies SA in collaboration with EIPHI Graduate School, ANR-17-EURE-0002.

\section{References}

[1] Z. Rigbi and L. Jilkén. The response of an elastomer filled with soft ferrite to mechanical and magnetic influences. Journal of magnetism and magnetic materials, 37(3):267-276, 1983.

[2] M.R. Jolly, J.D. Carlson, B.C. Muñoz, and T.A. Bullions. The magnetoviscoelastic response of elastomer composites consisting of ferrous particles embedded in a polymer matrix. Journal of Intelligent Material Systems and Structures, 7(6):613-622, 1996.

[3] H. Böse and R. Röder. Magnetorheological elastomers with high variability of their mechanical properties. Journal of physics: Conference series, 149(1):012090 6pp, 2009.

[4] M. Lokander and B. Stenberg. Performance of isotropic magnetorheological rubber materials. Polymer Testing, 22(3):245-251, 2003.

[5] J.M. Linke, D.Y. Borin, and S. Odenbach. First-order reversal curve analysis of magnetoactive elastomers. RSC Advances, 6(102):100407-100416, 2016.

[6] J. Sutrisno, A. Purwanto, and S.A. Mazlan. Recent progress on magnetorheological solids: materials, fabrication, testing, and applications. Advanced engineering materials, 17(5):563597, 2015.

[7] Q. Wen, Y. Wang, and X. Gong. The magnetic field dependent dynamic properties of magnetorheological elastomers based on hard magnetic particles. Smart Materials and Structures, 26(7):075012 (9p), 2017.

[8] L. Bodelot, J. P. Voropaieff, and T. Pössinger. Experimental investigation of the coupled magnetomechanical response in magnetorheological elastomers. Experimental Mechanics, 58(2):207-221, 2018.

[9] K. Danas, S.V. Kankanala, and N. Triantafyllidis. Experiments and modeling of iron-particle-filled magnetorheological elastomers. Journal of the Mechanics and Physics of Solids, 60(1):120-138, 2012 .

[10] M. Norouzi, M. Gilani, S.S. Alehashem, and H. Vatandoost. Dynamic characterization and modeling of isotropic magnetorheological elastomers under tensile-compressive loadings. IEEE Transactions on Magnetics, 53(9):1-12, 2017.

[11] G.V. Stepanov, A.V. Chertovich, and E.Y. Kramarenko. Magnetorheological and deformation properties of magnetically controlled elastomers with hard magnetic filler. M. Journal of Magnetism and Magnetic Materials, 324(21):3448-3451, 2012.

[12] D.Y. Borin, G.V. Stepanov, and S. Odenbach. Tuning the tensile modulus of magnetorheological elastomers with magnetically hard powder. Journal of Physics: Conference Series, 412(1):012040 (5pp), 2013.

[13] J.H. Koo, A Dawson, and H.J. Jung. Characterization of actuation properties of magnetorheological elastomers with embedded hard magnetic particles. Journal of Intelligent Material Systems and Structures, 23(9):1049-1054, 2012.

[14] E.Y. Kramarenko, A.V. Chertovich, G.V. Stepanov, A.S. Semisalova, L.A. Makarova, N.S. Perov, and A.R. Khokhlov. Magnetic and viscoelastic response of elastomers with hard magnetic filler. Smart materials and structures, 24(3):035022 (11p), 2015.

[15] M Schümann, D Yu Borin, S Huang, Günter K Auernhammer, R Müller, and S Odenbach. A characterisation of the magnetically induced movement of ndfeb-particles in magnetorheological elastomers. Smart Materials and Structures, 26(9):095018, 2017.

[16] G.V. Stepanov, D.Y. Borin, and P.A. Storozhenko. Rotation of magnetic particles inside the 
polymer matrix of magnetoactive elastomers with a hard magnetic filler. Journal of Magnetism and Magnetic Materials, 431:138-140, 2017.

[17] G.V. Stepanov, D.Y. Borin, E.Y. Kramarenko, V.V. Bogdanov, D.A. Semerenko, and P.A. Storozhenko. Magnetoactive elastomer based on magnetically hard filler: synthesis and study of viscoelastic and damping properties. Polymer science series A, 56(5):603-613, 2014.

[18] T. Szmidt, D. Pisarski, R. Konowrocki, S. Awietjan, and A. Boczkowska. Adaptive damping of a double-beam structure based on magnetorheological elastomer. Shock and Vibration, 2019:16, 2019.

[19] Y. Xia, M. Ruzzene, and A. Erturk. Dramatic bandwidth enhancement in nonlinear metastructures via bistable attachments. Applied Physics Letters, 114(9):093501, 2019.

[20] NuSil. Med-4014. https://nusil.com/product/med-4014_high-consistency-silicone-elastomer, accessed february 20, 2020.

[21] Y. Kim, H. Yuk, R. Zhao, S.A. Chester, and X. Zhao. Printing ferromagnetic domains for untethered fast-transforming soft materials. Nature, 558(7709):274-279, 2018.

[22] P. Małecki, M. Królewicz, J. Krzak, J. Kaleta, and J. Pigłowski. Dynamic mechanical analysis of magnetorheological composites containing silica-coated carbonyl iron powder. Journal of Intelligent Material Systems and Structures, 26(14):1899-1905, 2015.

[23] K.A. Kalina, J. Brummund, P. Metsch, M. Kästner, D.Y. Borin, J.M. Linke, and S. Odenbach. Modeling of magnetic hystereses in soft mres filled with ndfeb particles. Smart Materials and Structures, 26(10):105019 (12pp), 2017.

[24] P. Butaud, M. Ouisse, V. Placet, F. Renaud, T. Travaillot, A. Maynadier, and C. Rogueda-Berriet. Identification of the viscoelastic properties of the tba/pegdma polymer from multi-loading modes conducted over a wide frequency-temperature scale range. Polymer Testing, 69:250-258, 2018.

[25] F. Renaud, J.-L. Dion, G. Chevallier, I. Tawfiq, and R. Lemaire. A new identification method of viscoelastic behavior: Application to the generalized maxwell model. Mechanical Systems and Signal Processing, 25(3):991-1010, 2011.

[26] J. Fröhlich, W. Niedermeier, and H. D. Luginsland. The effect of filler--filler and filler-elastomer interaction on rubber reinforcement. Composites Part A: Applied Science and Manufacturing, 36(4):449-460, 2015.

[27] K. P. Menard. Dynamic Mechanical Analysis - A practical introduction. CRC Press, 1999.

[28] L. Mullins. Softening of rubber by deformation. rubber chemistry and technology. Journal of Physics: Condensed Matter, 42(1):339-362, 1969.

[29] A.R. Payne. The dynamic properties of carbon black-loaded natural rubber vulcanizates. part 1. Journal of applied polymer science, 6(19):57-63, 1962. 


\section{Appendix}

Table 3. Characteristics of samples for magnetic analysis

\begin{tabular}{|c|c|c|c|c|}
\hline Specimen & $\begin{array}{l}\Phi \\
{[\%]}\end{array}$ & $\begin{array}{l}w \\
{[\operatorname{mass} \%]}\end{array}$ & $\begin{array}{l}\text { diameter } \\
{[\mathrm{mm}]}\end{array}$ & $\begin{array}{l}\text { height } \\
{[\mathrm{mm}]}\end{array}$ \\
\hline$S_{m a g 1}$ & 29.8 & 75 & 5.80 & 0.85 \\
\hline$S_{\text {mag } 2}$ & 21.6 & 66 & 5.78 & 0.90 \\
\hline$S_{m a g 3}$ & 29.8 & 75 & 4.25 & 0.82 \\
\hline$S_{m a g 4}$ & 12.4 & 50 & 5.50 & 0.97 \\
\hline$S_{m a g 5}$ & 12.4 & 50 & 5.50 & 0.97 \\
\hline$S_{\text {mag6 }}$ & 12.4 & 50 & 5.50 & 0.97 \\
\hline$S_{m a g 7}$ & 21.6 & 66 & 5.50 & 0.97 \\
\hline$S_{m a g 8}$ & 21.6 & 66 & 5.50 & 0.97 \\
\hline$S_{\text {mag } 9}$ & 21.6 & 66 & 5.50 & 0.97 \\
\hline$S_{m a g 10}$ & 29.8 & 75 & 5.50 & 0.97 \\
\hline$S_{m a g 11}$ & 29.8 & 75 & 5.50 & 0.97 \\
\hline$S_{\operatorname{mag} 12}$ & 29.8 & 75 & 5.50 & 0.97 \\
\hline$S_{\operatorname{mag} 13}$ & 32.2 & 77 & 5.50 & 0.97 \\
\hline$S_{m a g 14}$ & 32.2 & 77 & 5.50 & 0.97 \\
\hline$S_{\operatorname{mag} 15}$ & 32.2 & 77 & 5.50 & 0.97 \\
\hline
\end{tabular}

Table 4. Characteristics of samples for mechanical analysis

\begin{tabular}{lrrlll}
\hline sample & $\begin{array}{r}\Phi \\
{[\%]}\end{array}$ & $\begin{array}{l}w \\
{[\mathrm{mass} \%]}\end{array}$ & $\begin{array}{l}L_{0} \\
{[\mathrm{~mm}]}\end{array}$ & $\begin{array}{l}h_{1} \\
{[\mathrm{~mm}]}\end{array}$ & $\begin{array}{l}h_{2} \\
{[\mathrm{~mm}]}\end{array}$ \\
\hline$S_{\text {mec } 1}$ & 0 & 0 & 15 & 18.5 & 2.5 \\
$S_{\text {mec } 2}$ & 19.4 & 63 & 15 & 18.3 & 2.5 \\
\hline
\end{tabular}


Table 5. Characteristics of sample for magneto-mechanical analysis

\begin{tabular}{|c|c|c|c|c|c|c|}
\hline sample & $\begin{array}{l}\Phi \\
{[\mathrm{vol} \%]}\end{array}$ & $\begin{array}{l}w \\
\text { [mass\%] }\end{array}$ & $\begin{array}{l}L_{0} \\
{[\mathrm{~mm}]}\end{array}$ & $h_{1}$ & $h_{2}$ & $\vec{M}$ \\
\hline \multicolumn{7}{|c|}{ before magnetization } \\
\hline$S_{m m 1}$ & 19.4 & 63 & 12.3012 .3312 .32 & 18 & 3.16 & - \\
\hline$S_{m m 2}$ & 19.4 & 63 & $\begin{array}{llll}12.41 & 12.33 & 12.23\end{array}$ & 18 & 3.14 & - \\
\hline$S_{m m 3}$ & 19.4 & 63 & 12.3212 .1212 .24 & 18 & 3.12 & - \\
\hline$S_{m m 4}$ & 19.4 & 63 & 12.4212 .2412 .37 & 18 & 3.11 & - \\
\hline$S_{m m 5}$ & 19.4 & 63 & 12.4912 .3312 .41 & 18 & 3.13 & - \\
\hline$S_{m m 6}$ & 19.4 & 63 & $12.2512 .31 \quad 12.39$ & 18 & 3.12 & - \\
\hline$S_{m m 7}$ & 19.4 & 63 & 12.3312 .3312 .29 & 18 & 3.12 & - \\
\hline$S_{m m 8}$ & 19.4 & 63 & 12.3112 .3112 .31 & 18 & 3.12 & - \\
\hline \multicolumn{7}{|c|}{ after magnetization } \\
\hline$S_{m m 1}$ & 19.4 & 63 & 12.2412 .3112 .31 & 18 & 3.16 & $\vec{M} \| L_{0}$ \\
\hline$S_{m m 2}$ & 19.4 & 63 & 12.3012 .3012 .16 & 18 & 3.14 & $\overrightarrow{\vec{M}} \| L_{0}$ \\
\hline$S_{m m 3}$ & 19.4 & 63 & $12.46 \quad 12.22 \quad 12.23$ & 18 & 3.13 & $\vec{M} \| L_{0}$ \\
\hline$S_{m m 4}$ & 19.4 & 63 & 12.3712 .3012 .24 & 18 & 3.12 & $\vec{M} \| L_{0}$ \\
\hline$S_{m m 5}$ & 19.4 & 63 & 12.2512 .2412 .32 & 18 & 3.12 & $\vec{M} \perp L_{0}$ \\
\hline$S_{m m 6}$ & 19.4 & 63 & 12.2512 .3012 .24 & 18 & 3.11 & $\vec{M} \perp L_{0}$ \\
\hline$S_{m m 7}$ & 19.4 & 63 & 12.3212 .2412 .31 & 18 & 3.12 & $\vec{M} \perp L_{0}$ \\
\hline$S_{m m 8}$ & 19.4 & 63 & 12.3212 .2512 .24 & 18 & 3.12 & $\vec{M} \perp L_{0}$ \\
\hline
\end{tabular}

